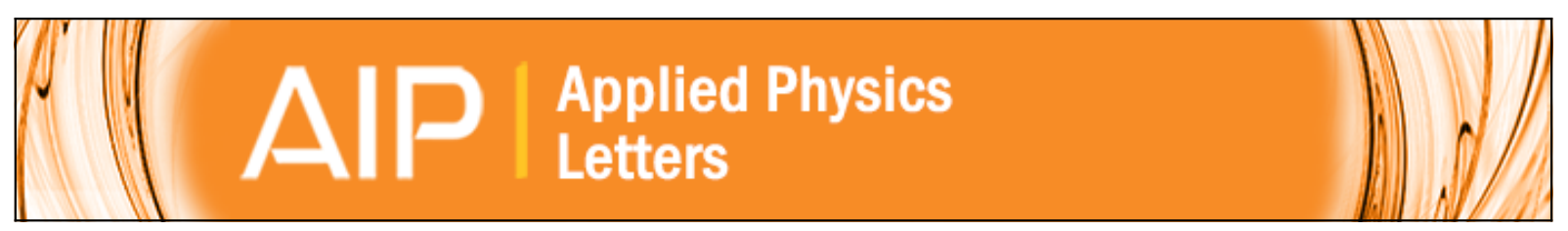

\title{
Enhancing the emission directionality of organic light-emitting diodes by using photonic microstructures
}

Shuyu Zhang, Graham A. Turnbull, and Ifor D. W. Samuel

Citation: Applied Physics Letters 103, 213302 (2013); doi: 10.1063/1.4829759

View online: http://dx.doi.org/10.1063/1.4829759

View Table of Contents: http://scitation.aip.org/content/aip/journal/apl/103/21 ?ver=pdfcov

Published by the AIP Publishing

\section{Articles you may be interested in}

Enhanced performance of organic light-emitting diodes using two-dimensional zinc sulfide photonic crystals

J. Appl. Phys. 106, 113105 (2009); 10.1063/1.3264886

Organic light-emitting diodes with photonic crystals on glass substrate fabricated by nanoimprint lithography Appl. Phys. Lett. 90, 111114 (2007); 10.1063/1.2713237

Enhanced light extraction efficiency from organic light emitting diodes by insertion of a two-dimensional photonic crystal structure

J. Appl. Phys. 96, 7629 (2004); 10.1063/1.1815049

Reduction of operating voltage in organic light-emitting diode by corrugated photonic crystal structure Appl. Phys. Lett. 85, 5769 (2004); 10.1063/1.1836867

A high-extraction-efficiency nanopatterned organic light-emitting diode Appl. Phys. Lett. 82, 3779 (2003); 10.1063/1.1577823

\section{AlP $\mid \begin{aligned} & \text { Journal of } \\ & \text { Applied Physics }\end{aligned}$}

Journal of Applied Physics is pleased to announce André Anders as its new Editor-in-Chief 


\title{
Enhancing the emission directionality of organic light-emitting diodes by using photonic microstructures
}

\author{
Shuyu Zhang, Graham A. Turnbull, ${ }^{\text {a) }}$ and Ifor D. W. Samuel ${ }^{\text {a) }}$ \\ Organic Semiconductor Centre, SUPA, School of Physics and Astronomy, University of St Andrews, \\ North Haugh, St Andrews, Fife KY16 9SS, United Kingdom
}

(Received 3 September 2013; accepted 28 October 2013; published online 21 November 2013)

\begin{abstract}
We report microstructured organic light-emitting diodes (OLEDs) with directional emission based on efficient solution-processable europium-OLEDs patterned by solvent assisted microcontact molding. The angle dependence of the light emission is characterized for OLEDs with square-array photonic crystals with periods between $275 \mathrm{~nm}$ and $335 \mathrm{~nm}$. The microstructured devices have emission patterns strongly modified from the Lambertian emission of planar OLEDs and can approximately double the emitted power in a desired angle range in both s- and p-polarizations. The modified emission is attributed to light diffracted out of the waveguide modes of the OLEDs. (C) 2013 AIP Publishing LLC. [http://dx.doi.org/10.1063/1.4829759]
\end{abstract}

In this paper, we explore the control of the emission direction of organic light-emitting diodes (OLEDs). Many aspects of OLEDs have been comprehensively studied and well controlled including power efficiency, chromaticity, and color stability, yet the directionality of the emission has received little attention. Directional emission could be useful for a range of application including biological sensors, ${ }^{1}$ autostereoscopic naked-eye 3D displays, ${ }^{2}$ and visible light communications. ${ }^{3}$ A powerful approach to modulate light emission and propagation is the use of photonic microstructures. These have been widely applied in OLEDs to increase efficiency through light extraction from the substrate mode, ${ }^{4-6}$ the waveguide mode, ${ }^{7-12}$ and/or the surface plasmon polariton (SPP) mode. ${ }^{13-15}$ However, very few papers have focused on trying to confine the emission power into a reduced range of angles. Tsutsui et al. ${ }^{16}$ introduced an optical resonant cavity with a dielectric reflector composed of $\mathrm{SiO}_{2} / \mathrm{TiO}_{2}$ bilayers into the OLED and strongly shaped the spatial distribution of the light emission. Feng et al. ${ }^{17}$ reported an approach of achieving directional emission by making a top-emitting microstructured OLED which out-coupled the SPP mode into the free space. Here we present a different approach to enhance the emission directionality by controlling the waveguide modes using embedded microstructures in the devices. The solution-processable europium(Eu)-based OLEDs ${ }^{18}$ are patterned using solvent assisted microcontact molding (SAMIM); ${ }^{19}$ a simple replication process which could be scaled for volume production.

Previously, we have developed a highly efficient solution-processable Eu-based OLED with commercially available materials, ${ }^{18}$ which exhibited an external quantum efficiency of $4.3 \%$ at a brightness of $100 \mathrm{Cd} / \mathrm{m}^{2}$. The microstructured Eu-based OLEDs in this work were fabricated in a very similar way. ITO-coated soda lime glass substrates were cleaned by ultrasound in acetone and 2-propanol, followed by an oxygen plasma treatment. A $40 \mathrm{~nm}$-thick Poly(3,4-ethylenedioxythiophene): poly(styrenesulfonate)

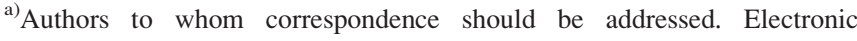
addresses: gat@st-andrews.ac.uk and idws@st-andrews.ac.uk.
}

(PEDOT:PSS) was spin-coated on the ITO and baked at $120^{\circ} \mathrm{C}$ for $10 \mathrm{~min}$. A hole-transport layer of $35 \mathrm{~nm}$-thick poly(N-vinylcarbazole) (PVK) was spin-coated on the PEDOT:PSS layer and baked at $80^{\circ} \mathrm{C}$ for $2 \mathrm{~h}$ in a nitrogen glove box. The remaining device fabrication steps were carried out in air. An emissive layer of $120 \mathrm{~nm}$-thick CBP:PBD:Eu(DBM) ${ }_{3} B$ phen $(5 \mathrm{wt} \%)$ was spin-coated on the PVK layer $\left(\mathrm{CBP}=4,4^{\prime}-\mathrm{N}, \mathrm{N}^{\prime}\right.$-dicarbazole-biphenyl; $\mathrm{PBD}=$ 2-(tert-butyl-phenyl)-5-biphenylyl-1,3,4-oxadiazole; $\quad \mathrm{DBM}=$ dibenzoylmethane). The emissive layer was then patterned by applying chlorobenzene to an elastomeric mold and pressing it onto the emissive layer. This process, known as SAMIM, requires no heat so it would not affect the optical properties of the patterned film. Using this technique, 2D pillar grating structures with four different grating periods of $275 \mathrm{~nm}, 290 \mathrm{~nm}, 305 \mathrm{~nm}$, and $335 \mathrm{~nm}$ were transferred reliably from the silicon master to the emissive layer with a groove depth of over $40 \mathrm{~nm}$. After the SAMIM process, the samples were transferred into a vacuum evaporation system where an electron-transport layer of $60 \mathrm{~nm}$-thick 1,3,5tris(2-N-phenylbenzimidazolyl)benzene (TPBI) was deposited through a shadow mask. A cathode of $\mathrm{LiF} / \mathrm{Al} / \mathrm{Ag}$ $(0.5 \mathrm{~nm} / 1 \mathrm{~nm} / 40 \mathrm{~nm})$ was then deposited on the TPBI layer in the same vacuum system. The ultra-thin LiF/Al bilayer acted as an effective electron injector and the $\mathrm{Ag}$ layer provides a high reflection and good conductivity. ${ }^{20}$ The external quantum efficiency of $\mathrm{LiF} / \mathrm{Al} / \mathrm{Ag}$-cathode planar devices is very close to that of $\mathrm{LiF} / \mathrm{Al}$-cathode ones. After the evaporation, the devices were encapsulated with optical curing adhesive (Norland NOA68) and glass coverslips in the glove box. The whole fabrication process is shown in Fig. 1(a).

The photonic microstructures formed by the SAMIM process were characterized by atomic force microscopy (AFM), which is shown in Fig. 1(b). Planar OLEDs without the SAMIM process were also made as references. The angular dependence of the emission from the OLEDs was measured using the apparatus in Fig. 1(c). The light emitted through the glass substrates of the OLEDs was collected by a fiber coupled Andor DV420-BV CCD spectrometer. The fiber collector was able to move in a plane perpendicular to 


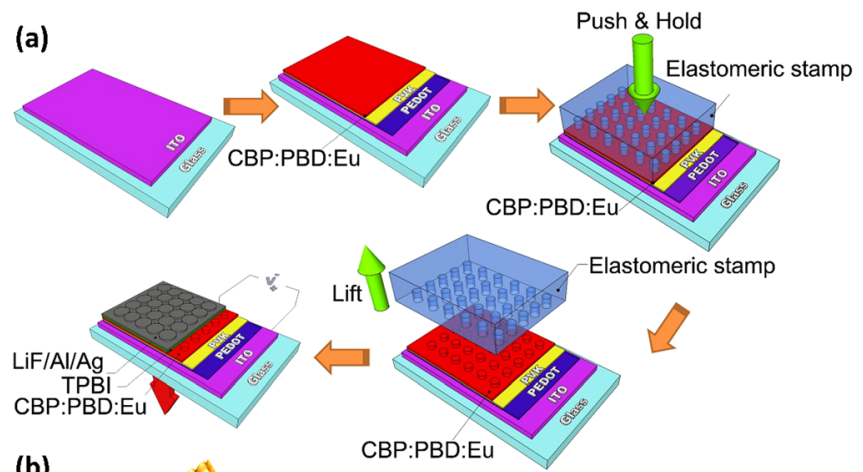

(b)

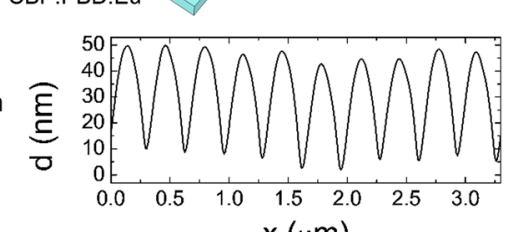

(c)
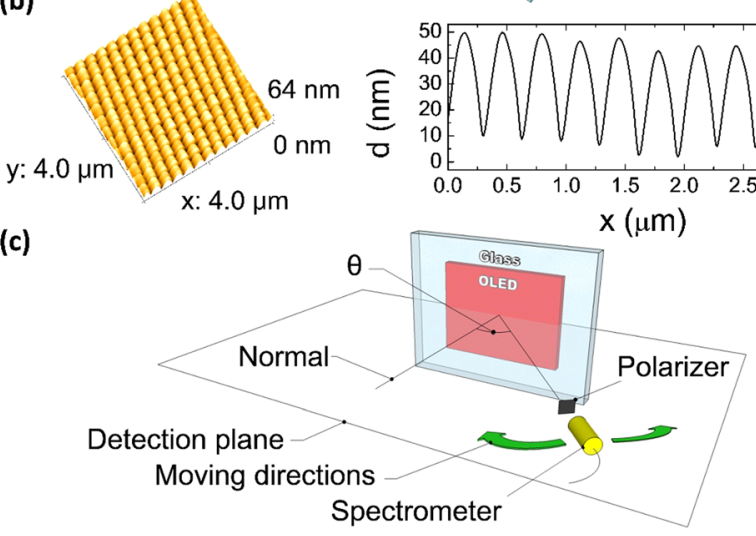

FIG. 1. (a) The fabrication process of the microstructured Eu-based OLEDs; (b) the AFM image of the patterned emissive layer; and (c) the measurement rig of the angular dependent emission profiles.

the substrates from the normal direction to an oblique angle up to $70^{\circ}$ with an interval of $2^{\circ}$. A polarizer was mounted in front of the fiber detector to record the emission profiles with different polarizations. The stability of the emission was checked after scanning; the OLEDs typically retained $>85 \%$ of the initial brightness during the measurement period.

Since the electroluminescence spectra of the Eu-based OLED devices at room temperature only have a dominant emission peak at $612 \mathrm{~nm}$ with a full-width-half-maximum of less than $5 \mathrm{~nm}$, the angular dependent emission profiles in the range of $609 \mathrm{~nm}$ to $614 \mathrm{~nm}$ were summed in order to identify the direction into which most of the emitted power flows. The results of these integrated angular dependent emission profiles are shown in Fig. 2. The unpatterned planar devices show an angular emission profile very close to Lambertian while the angular dependence of the microstructured OLEDs was very different. In the s-polarization, the peak power was detected at angles of $36^{\circ}, 28^{\circ}, 24^{\circ}$, and $10^{\circ}$ from the normal to the surface, corresponding to grating periods of $275 \mathrm{~nm}, 290 \mathrm{~nm}, 305 \mathrm{~nm}$, and $335 \mathrm{~nm}$, respectively. For the p-polarization, the corresponding peaks were observed at angles of $38^{\circ}, 30^{\circ}, 24^{\circ}$, and $14^{\circ}$. There is some emission at all angles due to the light which was directly emitted from the devices and not affected by the photonic

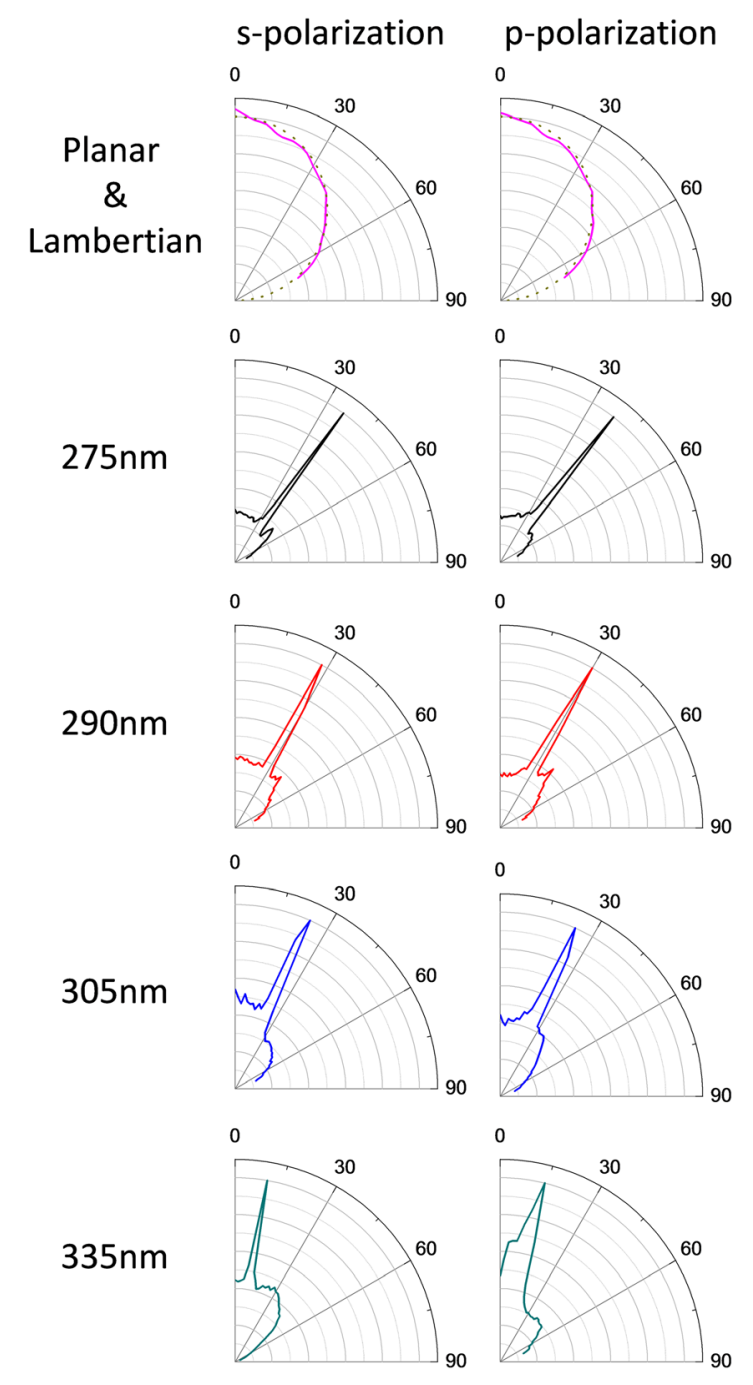

FIG. 2. The integrated angular dependent emission profiles in a range of $609 \mathrm{~nm}$ to $614 \mathrm{~nm}$ of the microstructured devices with grating periods of $275 \mathrm{~nm}, 290 \mathrm{~nm}, 305 \mathrm{~nm}$, and $335 \mathrm{~nm}$ as well as the unpatterned planar devices (in purple solid curve) and a Lambertian emitter (in dark yellow dots).

microstructures. To assess the increase in beam directionality, we define a parameter called the fraction of emission (FOE) given by the percentage of emitted power integrated in a specified range of angles in the detection plane. Table I shows a comparison of the FOE of microstructured Eu-based OLEDs with that of a Lambertian emitter in both polarizations. The angle range is $4^{\circ}$, centered at the angle of peak emission from the microstructured OLEDs. A Lambertian emitter shows a FOE of around $8 \%$ to $10 \%$, whereas the microstructured devices could improve the FOE to $16 \%$ to $19 \%$ in s-polarization and $18 \%$ to $20 \%$ in p-polarization. This means the microstructured devices can double the emitted power in the same angular range.

The beaming direction is determined by both the effective refractive index of the OLED and the grating period of the

TABLE I. Comparison of the FOE in the detection plane of microstructured Eu-based OLEDs with that of a Lambertian emitter.

\begin{tabular}{|c|c|c|c|c|}
\hline Grating periods & $275 \mathrm{~nm}$ & $290 \mathrm{~nm}$ & $305 \mathrm{~nm}$ & $335 \mathrm{~nm}$ \\
\hline FOE of the microstructured OLEDs in s-polarization & $19 \%\left(34^{\circ}-38^{\circ}\right)$ & $19 \%\left(26^{\circ}-30^{\circ}\right)$ & $18 \%\left(22^{\circ}-26^{\circ}\right)$ & $16 \%\left(8^{\circ}-12^{\circ}\right)$ \\
\hline FOE of the microstructured OLEDs in p-polarization & $20 \%\left(36^{\circ}-40^{\circ}\right)$ & $19 \%\left(28^{\circ}-32^{\circ}\right)$ & $19 \%\left(22^{\circ}-26^{\circ}\right)$ & $18 \%\left(12^{\circ}-16^{\circ}\right)$ \\
\hline FOE of a Lambertian emitter & $8 \%$ & $9 \%$ & $9 \%$ & $10 \%$ \\
\hline
\end{tabular}


patterned photonic microstructure. COMSOL Multiphysics v4.3 was used to model the waveguide modes and the SPP mode of the OLED. The simulations were carried out for an unpatterned planar device. The modes were calculated by finding the in-plane propagation vector for which the fields dropped off exponentially outside the waveguide. The corresponding effective refractive indices were then calculated. Figs. 3(a) and 3(b) show the normalized intensity profiles of the supported modes in s- and p-polarizations and the real part of the refractive indices of all materials. In s-polarization, there is only one supported TE mode. In p-polarization, two modes are supported: a SPP mode (black dots in Fig. 3(b)) and a further TM mode (the red solid curve). These correspond to the coupled eigenmodes of a dielectric waveguide and a metal film. ${ }^{21}$ The calculated real part of the effective refractive index of the TE, TM, and SPP mode is 1.6548, 1.5717, and 1.8259, respectively. According to Bragg scattering, the in-plane wave vector components after the scattering

(a)

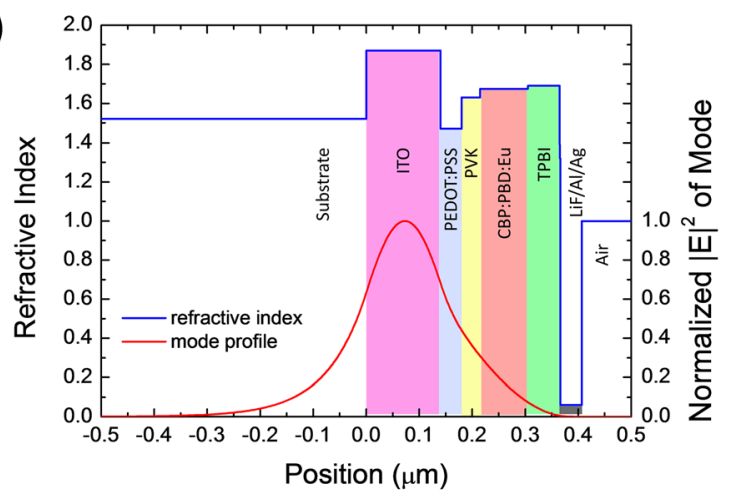

(b)

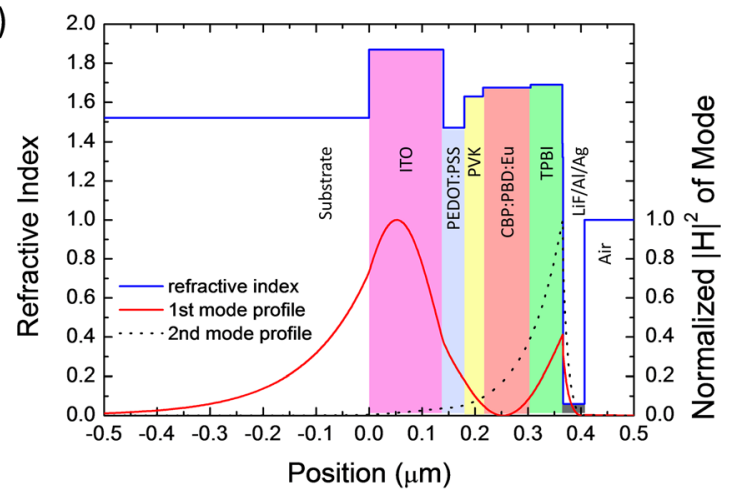

(c)

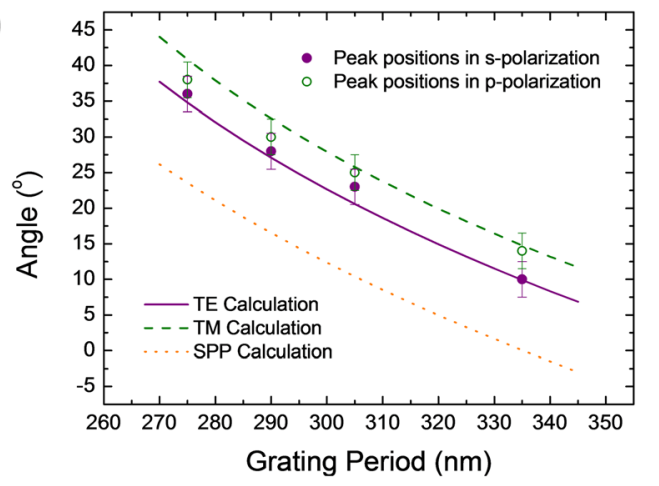

FIG. 3. The normalized intensity profiles of the supported modes in (a) spolarization and (b) p-polarization and the real part of refractive indices of all materials; (c) the experimentally measured (in circles) and simulated (in curves) angles of peak emission from the microstructured devices as a function of the grating periods. $\mathrm{k}_{/ /}^{\prime}$ was determined by the difference between the in-plane wave vector components before the scattering $\mathrm{k}_{/ \text {! }}$ and the grating vector $\mathbf{G}(\mathrm{G}=2 \pi / \Lambda, \Lambda$ is the grating period) which is shown in Formula (1)

$$
\mathrm{k}_{/ /}^{\prime}=\mathrm{k}_{/ /}-\mathrm{mG}=\beta-\mathrm{mG}
$$

where $\mathrm{m}$ is an integer number specifying the scattering order and $\beta$ is the propagation constant of the waveguide modes. The out-coupling angle of a certain mode then can be expressed as

$$
\theta\left(\lambda_{0}, \Lambda\right)=\arcsin \left[\operatorname{Re}\left(\mathrm{n}_{\mathrm{eff}}\right)-\mathrm{m} \lambda_{0} / \Lambda\right]
$$

Here $\mathrm{n}_{\text {eff }}$ is the effective refractive index $\left(\mathrm{n}_{\mathrm{eff}}=\lambda_{0} \beta / 2 \pi\right)$ and $\lambda_{0}$ is the vacuum wavelength. So, for example, if the grating period of the patterned photonic microstructure is $335 \mathrm{~nm}$ and the free space wavelength is $612 \mathrm{~nm}$, only one scattered order $\mathrm{m}=1$ is able to be out-coupled by Bragg scattering and the emission is predicted to be strongly peaked at $9.9^{\circ}, 14.8^{\circ}$, and $0.1^{\circ}$ for TE, TM, and SPP modes by calculation. The experimental results show the emission peaked at $10^{\circ}$ and $14^{\circ}$ which match well with the simulation results of TE and TM modes. Fig. 3(c) shows the experimentally measured and simulated angles of peak emission from the microstructured devices as a function of the grating periods. The experimental and simulation results are in good agreement, which indicates the emission peaks observed can be attributed to the out-coupling of the TE and TM modes. Interestingly, we did not observe any noticeable emission due to the out-coupling of the SPP mode through the substrate, but a peak corresponding to the SPP mode was observed in top emission through the metal film.

In summary, we have developed solution-processable OLEDs with directional emission by patterning photonic microstructures into the emissive layer. The microstructured devices strongly boost the emitted power in a desired angle range in both $\mathrm{s}$ - and p-polarizations and the fraction of emission could be doubled. The patterning process is simple and effective, offering the potential for directional OLEDs to be volume produced by nano-replication.

S. Zhang, G. A. Turnbull, and I. D. W. Samuel are grateful to the Scottish Universities Physics Alliance (SUPA) and the Engineering and Physical Sciences Research Council (EPSRC) for financial support. The authors would also like to thank Dr. Georgios Tsiminis for the design of the silicon master structures and thank Dr. Yue Wang for the help with the fabrication of the elastomeric molds.

${ }^{1}$ M. Ramuz, D. Leuenberger, and L. Burgi, J. Polym. Sci., Part B: Polym. Phys. 49(1), 80-87 (2011).

${ }^{2}$ D. Fattal, Z. Peng, T. Tran, S. Vo, M. Fiorentino, J. Brug, and R. G. Beausoleil, Nature 495(7441), 348-351 (2013).

${ }^{3}$ H. Elgala, R. Mesleh, and H. Haas, IEEE Commun. Mag. 49(9), 56-62 (2011).

${ }^{4}$ Y. Sun and S. R. Forrest, J. Appl. Phys. 100(7), 073106 (2006).

${ }^{5}$ S. Jeon, J. W. Kang, H. D. Park, J. J. Kim, J. R. Youn, J. Shim, J. H. Jeong, D. G. Choi, K. D. Kim, A. O. Altun, S. H. Kim, and Y. H. Lee, Appl. Phys. Lett. 92(22), 223307 (2008). 
${ }^{6}$ J.-B. Kim, J.-H. Lee, C.-K. Moon, S.-Y. Kim, and J.-J. Kim, Adv. Mater. 25(26), 3571-3577 (2013).

${ }^{7}$ J. M. Ziebarth, A. K. Saafir, S. Fan, and M. D. McGehee, Adv. Funct. Mater. 14(5), 451-456 (2004).

${ }^{8}$ W. H. Koo, S. M. Jeong, F. Araoka, K. Ishikawa, S. Nishimura, T. Toyooka, and H. Takezoe, Nat. Photonics 4(4), 222-226 (2010).

${ }^{9}$ X.-L. Zhang, J. Feng, J.-F. Song, X.-B. Li, and H.-B. Sun, Opt. Lett. 36(19), 3915-3917 (2011).

${ }^{10}$ J. Hauss, T. Bocksrocker, B. Riedel, U. Lemmer, and M. Gerken, Opt. Express 19(14), A851-A858 (2011).

${ }^{11}$ T. Bocksrocker, F. Maier-Flaig, C. Eschenbaum, and U. Lemmer, Opt. Express 20(6), 6170-6174 (2012).

${ }^{12}$ W. H. Koo, W. Youn, P. F. Zhu, X. H. Li, N. Tansu, and F. So, Adv. Funct. Mater. 22(16), 3454-3459 (2012).

${ }^{13}$ J. M. Lupton, B. J. Matterson, I. D. W. Samuel, M. J. Jory, and W. L. Barnes, Appl. Phys. Lett. 77(21), 3340-3342 (2000).
${ }^{14}$ B. J. Matterson, J. M. Lupton, A. F. Safonov, M. G. Salt, W. L. Barnes, and I. D. W. Samuel, Adv. Mater. 13(2), 123-127 (2001).

${ }^{15}$ C. J. Yates, I. D. W. Samuel, P. L. Burn, S. Wedge, and W. L. Barnes, Appl. Phys. Lett. 88(16), 161105 (2006).

${ }^{16}$ T. Tsutsui, N. Takada, S. Saito, and E. Ogino, Appl. Phys. Lett. 65(15), 1868-1870 (1994).

${ }^{17}$ J. Feng, T. Okamoto, and S. Kawata, Appl. Phys. Lett. 87(24) 241109 (2005).

${ }^{18}$ S. Zhang, G. A. Turnbull, and I. D. W. Samuel, Org. Electron. 13(12), 3091-3096 (2012).

${ }^{19}$ J. R. Lawrence, G. A. Turnbull, and I. D. W. Samuel, Appl. Phys. Lett. 82(23), 4023-4025 (2003).

${ }^{20}$ L. S. Hung, C. W. Tang, M. G. Mason, P. Raychaudhuri, and J. Madathil, Appl. Phys. Lett. 78(4), 544-546 (2001).

${ }^{21}$ M. L. Nesterov, A. V. Kats, and S. K. Turitsyn, Opt. Express 16(25), 20227-20240 (2008). 